\title{
Nursing Cooperation in a Patient with Superior Vena Cava Aneurysm Undergoing Contrast-Enhanced CT Examination: A Case Report
}

\author{
Shaofang Xian ${ }^{1}$, Li Hairui ${ }^{2, *}$ \\ ${ }^{1}$ Radiology Department, The First Affiliated Hospital of Jinan University, Guangzhou, China \\ ${ }^{2}$ Department of Cardiology, The First Affiliated Hospital of Jinan University, Guangzhou, China \\ Email address: \\ 495673101@qq.com (Shaofang Xian),jnuhenry@qq.com (Li Hairui) \\ ${ }^{*}$ Corresponding author
}

To cite this article:

Shaofang Xian, Li Hairui. Nursing Cooperation in a Patient with Superior Vena Cava Aneurysm Undergoing Contrast-Enhanced CT Examination: A Case Report. American Journal of Nursing Science. Vol. 10, No. 2, 2021, pp. 124-127. doi: 10.11648/j.ajns.20211002.12

Received: February 3, 2021; Accepted: February 26, 2021; Published: March 4, 2021

\begin{abstract}
Superior vena cava aneurysm is a rare life-threatening intrathoracic vascular lesion. Contrast-enhanced CT examination plays an important role for surgical planning. It needs to choose the appropriate vein as the puncture site for contrast agent injection. Hereby we report a 22-year-old male presented with sudden-onset dyspnea and unconsciousness for 2 hours and suffered from cardiac arrest before visiting our hospital. The patient's condition is critically ill, and the risk of contrast agent extravasation and aneurysm explosion is high. We established a multi-disciplinary team, involving Emergency Department, Cardiac Surgery Department, Radiological Department and intravenous therapy experts, for the integrated assessment of patients. And we activate the emergency cooperation protocol for critically ill patients. The superior vena cava can't be used for contrast injection. Anatomically, the femoral vein drains blood back to the heart through the inferior vena cava, which could avoid the risk of rupture of the superior vena cava aneurysm due to excessive pressure of bolus injection of contrast agent. The indwelling of femoral vein puncture can be used in the treatment of critically ill patients. The vital signs of patients were closely observed during the examination process. No contrast agent extravasation and allergy reaction was observed and CT images were clear. The effective nursing cooperation in this case ensured the safety and effectiveness of the examination, and laid the foundation for further treatment.
\end{abstract}

Keywords: Superior Vena Cava Aneurysm, Femoral Vein, Contrast-Enhanced CT, Nursing Cooperation

\section{Introduction}

Superior vena cava aneurysm, whose symptoms include chest pain, cough, expectoration, and shortness of breath on exertion, is a rare intrathoracic vascular lesion [1]. Its radiological manifestations are similar to mediastinal tumors on conventional on plain chest film [2]. Since its symptoms and signs aren't obvious and non-specific, and its etiology is still unknown, superior vena cava aneurysm tends to be misdiagnosed in early stage [3]. However, it has a great impact on patients' health due to its serious complications, such as rupture, thrombosis, and pulmonary embolism [4].

As a noninvasive examination tool, CT could clearly show the range, size, morphology and thrombus of the superior vena cava [5]. In our case, a critically ill patient is considered to be with a huge superior vena cava aneurysm. Contrast-enhanced CT examination plays an important role for surgical planning. In order to obtain clear image, it is necessary to intravenously inject contrast agent in the process of CT scanning. Therefore, it needs to choose the appropriate vein as the puncture site [6].

Internal jugular vein or subclavian vein catheterization is the common chosen route for contrast medium injection [7]. However, both these two veins drained through the superior vena cava back to the right atrium. The power injection of contrast agent, which is required to optimize the imaging quality [8], may induce the rupture or extravasation of contrast agent into thoracic cavity and cause life-threatening complication. In addition, internal jugular vein or subclavian vein catheterization may cause 
catheter related complications [9], among which venous perforation is the most dangerous one, especially in a patient with superior vena cava aneurysm. The femoral vein drained back to the heart through the inferior cava; therefore, femoral vein catheterization may prevent the rupture of superior vena cava aneurysm. After multi-disciplinary cooperation and careful nursing cooperation, the femoral vein was chosen as the route for contrast injection, which had not been reported in previous literatures, so that the patient could undergo contrasted-enhanced CT examination effectively and successfully. The details of our case report were as follows.

\section{Case Report}

\subsection{General Information}

A 22-year-old male patient, presented with sudden-onset dyspnea and unconsciousness for 2 hours, was admitted in Zhuhai Hospital of Integrated Traditional Chinese and Western Medicine on June 21, 2020. Due the unsatisfactory treatment effect, he was transferred to our hospital for further treatment on June 23. During the transfer, he suffered from sudden cardiac arrest. He was given cardiopulmonary resuscitation for about 5 minutes and then regained a pulse and spontaneous respirations. According to the examination result of Zhuhai Hospital of Integrated Traditional Chinese and Western Medicine, he was suspicious of having a space-occupying lesion in the right upper anterior and middle mediastinum. Considering the possibility of superior vena cava aneurysm, he was planned to undergo emergency CT examination to make a definite diagnosis. When the patient was in critical condition, the cooperation program for critical patients was activated immediately, and the medical technician and nursing team cooperated with each other. The patient had no special past medical history before this illness.

\subsection{Examination Protocols}

CTA is the most important examination in spiral CT imaging, which requires injection of 60-80 ml iodine contrast agent intravenously. The acquisition of volume data in target vessel is completed within tens of seconds after the examinee inflates at a time. All the information was obtained at the peak of contrast agent infusion, which provides a non-invasive examination method for the diagnosis of superior vena cava aneurysm, and displays planar and three-dimensional images [10]. Routine preparation of contrast CTA needs the establishment of peripheral venous access for the patients, C0068oose the straight vein with large caliber and good elasticity of upper limb, such as the three major veins in elbow and radial vein, and if necessary, use the external jugular vein. However, based on the consideration of the patient's condition and the risk that the high pressure injection pressure may cause the rupture of the aneurysm, it is not recommended to use the upper limb vessels for venipuncture. The discussion of results of the medical technician and nursing team: the femoral vein was selected and inserted an indwelling tube to inject contrast agent for the CTA scanning. Under the close observation and joint efforts of medical and nursing team, the CT enhancement examination of patients was successfully and effectively completed, and the stable and real functional imaging was also obtained. The image quality provided a foundation for the reliable and accurate surgical plans of the patients. The patient arrived at the CT room of Radiological Department at 22:25 and the examination was completed at 23:30 successfully.

\section{Key Points in Nursing}

\subsection{Activate the Emergency Plan and Choose the Appropriate Examination Scheme with the Cooperation of Doctor, Technician and Nursing Teams}

In contrast-enhanced $\mathrm{CT}$ examination, the dorsal hand veins and forearm veins were routinely chosen for bolus injection of contrast agent. Due to the special condition of the patient, the routine scheme couldn't be implemented. A multi-disciplinary cooperation team, involving Emergency Department, Cardiac Surgery Department, Radiological Department and intravenous therapy experts, was established to discuss the condition and select the appropriate scanning scheme. According to the patient's special condition, the superior vena cava can't be used for contrast injection, so the inferior vena cava was chosen. Anatomically, the femoral vein drains blood back to the heart through the inferior vena cava, which could avoid the risk of rupture of the superior vena cava aneurysm due to excessive pressure of bolus injection of contrast agent. The indwelling of femoral vein puncture can be used in the treatment of critically ill patients, which helps to save lives [11]. Previous study shown that it is a safe, reliable and feasible method to choose femoral vein as puncture site for intravenous bolus injection by hand push during contrast-enhanced CT scanning [12]. Based on the clinical experience of our multi-disciplinary team, preliminary injection of normal saline was used to evaluate the tolerance of femoral vein to high pressure during bolus of contrast agent, and the CT examination was successfully undergone for the patient.

\subsection{Closely Monitor the Vital Signs to Ensure the Safety of the Patient}

When the scanning protocol was determined, the nurses quickly assess whether there are metal objects in the neck and chest area of the patients. If there are metal buttons and zippers, don't take off the clothes at will, please remove them with scissors to avoid secondary injury. Food and drug allergy history, as well as any history of hyperthyroidism and abnormal renal function, should be taken from the patients and their family members. Contraindications should be excluded and written informed consent should be obtained. Connect the high-pressure syringe, fix it and then activate it 
for contrast agent injection. Properly fix all the tubes: place a small pillow at the junction between the endotracheal tube and the ventilator circuit to avoid the tubes falling off; place the gastric tube on the top of the skull and next to the ear; ensure that the fixing tape of the indwelling catheter of radial artery does not curl and fall off, and if necessary, replace the fixing tape with a new one; the urinary tube shold be clamped and fixed at lower limbs to avoid affecting the image quality and falling off the examination table; the competence of all the pipes should be ascertained by two people. The examination table is narrow and small, and the patient should be fixed with a restraint belt to avoid falling off, and if necessary, the patient should be accompanied by the family members [13]. The temperature in CT room is low so pay attention to keep warm. All the articles in the first-aid kit should be routine checked and sputum suction devices should be prepared. During the process of high pressure injection, closely observe the vital signs of patients, pay attention to the adverse drug reactions, if it happens, immediately stop the injection and give the appropriate treatment [14-15].

\subsection{Preventing Complications and Lowering the Risk of Contrast Agent Extravasation}

After confirming the position of indwelling catheter in femoral vein, $20 \mathrm{ml}$ normal saline was injected via the high-pressure syringe at a speed of $7 \mathrm{ml}$ to make sure the safety of iodine contrast agent injection. Then connect high-pressure syringe and fix it with elastic tape. Observe the injection pressure curve closely during the injection process, and stop the injection in case of any abnormality observed. After the examination, check whether there is fluid extravasation in the femoral vein, after confirming the imaging quality, separate the high-pressure syringe with the connecting tube, and explain the precautions to the doctor. Transfer the patient from the examination bed carefully by cooperating with several persons, check all indwelling tubes and make sure all of them fixed properly. Since the patient was on critical condition, explain to the doctor in charge to observe the patient closely within 30 minutes, and pay attention to whether there is delayed iodine contrast agent allergic reaction.

\subsection{Implementing Psychological Intervention and Relieving the Anxiety of Family Members}

The patient was in a coma when he entered the CT room; besides, the young patient was suffering from a serious disease with sudden onset and of unknown causes; furthermore, the treatment plan was unknown. His family members were in a period of fear and pessimism, therefore, the nurses took the initiative to carry out the relevant health education on the family members, mainly explaining the purpose and risk of the examination, and obtaining the understanding and cooperation of the family members. After the completion of the examination, the nurses actively inform the family members of the successful completion of the examination to enhance their confidence in treatment.

\section{Conclusion}

The CT examination showed a huge tumor-like protrusion, about $10 \mathrm{~cm} \times 6.4 \mathrm{~cm} \times 11.4 \mathrm{~cm}$ in diameter, in the superior vena cava of the patient which protruded to the right chest. The patient's is on critically ill condition. It is critical to establish a multi-disciplinary cooperation team urgently and select an appropriate scanning scheme to ensure the safety and successful completion of the examination. The evaluation of rare cases needs multi-team cooperation to evaluate whether the condition is suitable for CT enhancement examination and reduce the risk of examination. The effective nursing cooperation in this case ensured the safety and effectiveness of the examination, and laid the foundation for further treatment.

\section{References}

[1] Morales MM, Anacleto A, Ferreira Leal JC, et al. Saccular Superior Vena Cava Aneurysm: Case Report and Comprehensive Review. Ann Vasc Surg. 2020 Dec 14: S0890-5096(20)31021-9.

[2] Nunnelee JD. Superior vena cava syndrome. J Vasc Nurs. 2007 Mar; 25 (1): 2-5; quiz 6.

[3] Nakajima $Y$, Minami T, Ishigaki H, et al. A superior vena cava aneurysm discovered by chance at regular physical examination. Respir Investig. 2020 Jul; 58 (4): 291-294.

[4] Nair AA, Christopher DJ, Moidu F, et al. Superior vena caval obstruction: a rare presentation of Behcet's disease. BMJ Case Rep. 2020 Dec 17; 13 (12): e236658.

[5] Soares Souza LV, Souza AS, et al. Superior vena cava aneurysm: an unusual mediastinal mass. Eur J Cardiothorac Surg. 2021 Jan 4; 59 (1): 276-277.

[6] Sonavane SK, Milner DM, Singh SP, et al. Comprehensive Imaging Review of the Superior Vena Cava. Radiographics. 2015 Nov-Dec; 35 (7): 1873-92.

[7] Tan PY, Tham WP, Yong YR. Clinics in diagnostic imaging (194). SVC aneurysm. Singapore Med J. 2019 Jan; 60 (1): 17-21.

[8] Witting MD, Moayedi S, Dunning K, et al. Power Injection Through Ultrasound-Guided Intravenous Lines: Safety and Efficacy Under an Institutional Protocol. J Emerg Med. 2017 Jan; 52 (1): 16-22.

[9] Sánchez Matás C, Manresa Manresa F, Andrades Sardiña D, et al. Thoracoscopic approach of iatrogenic superior vena cava perforation after dialysis catheter placement. Cir Esp. 2020 Jun-Jul; 98 (6): 364-366.

[10] Zhang HY, Lian WS, Chai DZ. Large asymptomatic superior vena cava aneurysm. J Vasc Surg Venous Lymphat Disord. 2019 Jul; 7 (4): 591.

[11] Safety Committee of Japanese Society of Anesthesiologists. Practical guide for safe central venous catheterization and management 2017. J Anesth. 2020 Apr; 34 (2): 167-186. 
[12] Saugel B, Umgelter A, Schuster T, et al. Transpulmonary thermodilution using femoral indicator injection: a prospective trial in patients with a femoral and a jugular central venous catheter. Crit Care. 2010; 14 (3): R95. doi: 10.1186/cc9030.

[13] Schoenfeld AJ, Beck AW, Harris MB, et al. Evaluating the Cervical Spine in the Blunt Trauma Patient. J Am Acad Orthop Surg. 2019 Sep 1; 27 (17): 633-641.
[14] Chen Z, Ishizuka O, Imamura $T$, et al. Role of alpha1-adrenergic receptors in detrusor overactivity induced by cold stress in conscious rats. Neurourol Urodyn. 2009; 28 (3): 251-6.

[15] Park HJ, Son JH, Kim TB, et al. Relationship between Lower Dose and Injection Speed of Iodinated ContrastMaterial for CT and Acute Hypersensitivity Reactions: An Observational Study. Radiology. 2019 Dec; 293 (3): 565-572. 